\title{
Transparent UWB Antenna with IZTO/Ag/IZTO Multilayer Electrode Film
}

\author{
Seungman Hong, Youngsung Kim, and Chang Won Jung \\ Graduate School of Nano IT Design Fusion, Seoul National University of Science and Technology, 232 Gongneung-ro, \\ Nowon-gu, Seoul 139-743, Republic of Korea \\ Correspondence should be addressed to Chang Won Jung; changwoj@snut.ac.kr
}

Received 22 August 2016; Accepted 25 September 2016

Academic Editor: Herve Aubert

Copyright (c) 2016 Seungman Hong et al. This is an open access article distributed under the Creative Commons Attribution License, which permits unrestricted use, distribution, and reproduction in any medium, provided the original work is properly cited.

\begin{abstract}
Ultra-wide band (UWB) antennas with transparent indium zinc tin oxide (IZTO)/Ag/IZTO multilayer electrode films are presented. The geometry of the radiator of the proposed UWB antenna is a simple semicircular shape, and acryl is used for the transparent substrate. Three types of proposed antennas are used for the comparison: first, an UWB antenna with a copper sheet only (CUWB); second, a transparent UWB antenna with a transparent radiator only (TRUWB); and, third, an entirely transparent UWB antenna (TUWB). The IZTO/Ag/IZTO multilayer electrode is fabricated by a physical vapor deposition process in the highly maintained vacuum. The transmittance of the multilayer film at $550 \mathrm{~nm}$ wavelength is over $80 \%$, and the conductivity is $3,960,000 \mathrm{~S} / \mathrm{m}$. The proposed CUWB, TRUWB, and TUWB have peak gains of $4.17,3.14$, and $0.74 \mathrm{dBi}$ and radiation efficiency levels of $84.8,64.5$, and $42.1 \%$ at the center frequency of UWB service $(6.5 \mathrm{GHz})$, respectively.
\end{abstract}

\section{Introduction}

Transparent conducting oxide (TCO) is a material that is transparent and conductive [1-9]. TCO is a widely used material for products in the display industry [1], such as smartphone touch screen panels and organic light emitting diodes (OLED) [2]. TCO is a material composed of indium, zinc, tin, and oxidized materials [1-11]. Indium tin oxide (ITO), one of the TCOs, is mainly utilized in the display industry [3]. However, ITO is brittle because of its crystalline structure [4]. Thus, ITO needs to be strengthened. There are several solutions for this problem. One is fabricating ITO at a higher temperature, since ITO has an amorphous structure and has tolerance when it is fabricated at $200^{\circ} \mathrm{C}$ [5]. The other is depositing a nanoscale thickness ITO layer, since it has a higher bending radius than that of a thicker one [6]. However, the thinner ITO films have high sheet resistance and cannot be used for transparent antennas [7]. Because of low conductivity in the thinner ITO films, other ITO representatives, such as fluorine-doped tin oxide
(FTO) and aluminum zinc oxide (AZO), have been studied recently [8]. However, FTO and AZO still have relatively lower conductivity levels for practical applications [8]. For this reason, we used another representative, a sandwichtype multilayer electrode film that is composed of three layers of IZTO/Ag/IZTO. The IZTO/Ag/IZTO multilayer electrode film has an oxide/metal/oxide structure and enough conductivity to be used for passive devices, such as antennas [9-11].

In this paper, the performance levels of the transparent UWB (ultra-wide band) antennas are measured to check the feasibility of the transparent antennas using IZTO/Ag/IZTO multilayer electrode film. For the comparison of the transparent antennas in the UWB range, the simplest UWB antenna design is used [12]. We simulated, fabricated, and measured transparent UWB antennas using optimized multilayer electrode film with IZTO/Ag/IZTO, which is fabricated by the physical vapor deposition (PVD) process. Three types of antennas are designed: first, an UWB antenna using a copper sheet (CUWB); second, a transparent radiator only UWB 


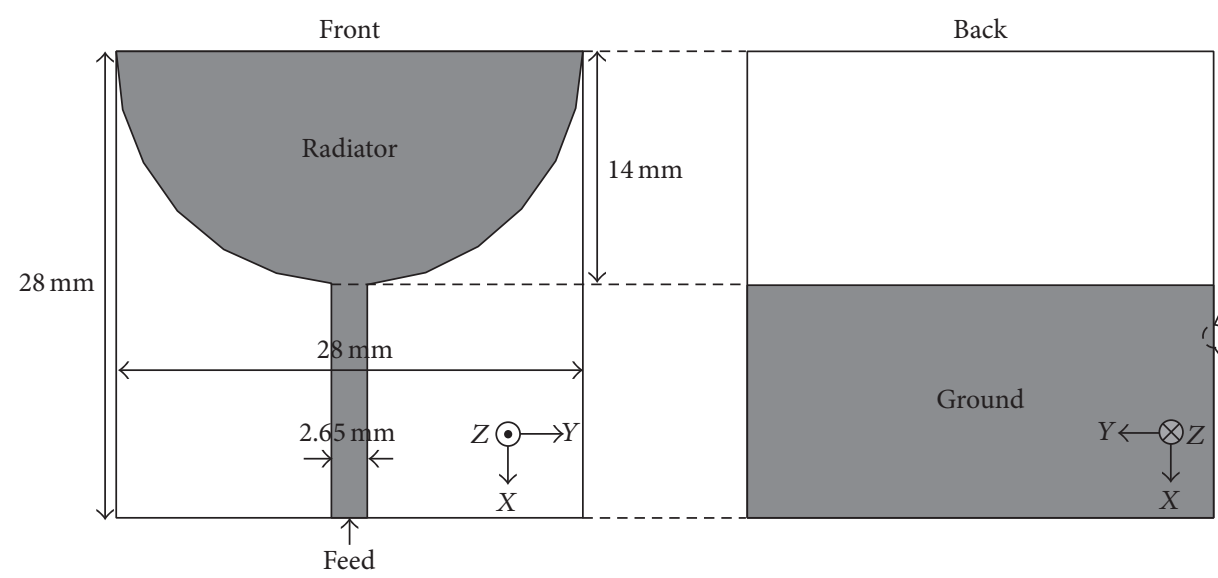

(a) (b)

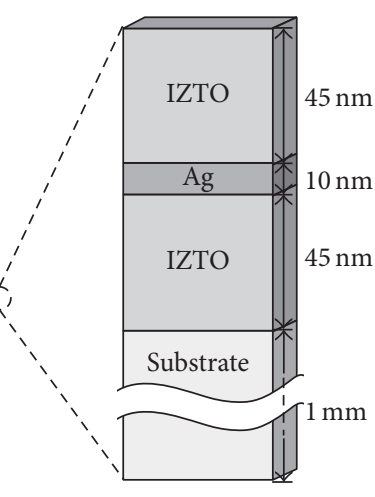

(c)

Figure 1: Design and dimension of the UWB antenna. (a) Front view. (b) Back view. (c) Structure of IZTO/Ag/IZTO multilayer electrode film and substrate.

antenna using an IZTO/Ag/IZTO radiator with a copper microstripline and ground plane (TRUWB); and, third, an entirely transparent UWB antenna (TUWB). CUWB is fabricated to compare the performances with the proposed transparent UWB antennas. TRUWB is designed for the case of mobile devices using a copper ground plane and microstripline with only a transparent radiator. TUWB is fabricated for entirely transparent devices that are available for wireless communication. The fabricated transparent antenna has higher performance than previous transparent UWB antenna which has gain of $0 \mathrm{~dB}[13]$.

\section{Antenna Design and Dimension}

The design and dimensions of the UWB antenna are depicted in Figure 1. The antenna shape is characterized by a semicircular radiator on the microstripline. The ground plane covers half of the back side of the substrate. A transparent acryl substrate $\left(\varepsilon_{r}=2.81\right.$ and tangent $\left.\delta=0.01\right)$ is employed for the proposed transparent UWB antennas. The size of the substrate is $28 \mathrm{~mm} \times 28 \mathrm{~mm} \times 1 \mathrm{~mm}$. For the transparent antennas (TRUWB and TUWB), IZTO/Ag/IZTO multilayer electrode film is used. The structure of the transparent IZTO/Ag/IZTO multilayer electrode on the substrate is depicted in Figure 1(c). As shown in the figure, the thickness of IZTO is $45 \mathrm{~nm}$ and that of silver is $10 \mathrm{~nm}$. The antennas are fabricated with three types, which are shown in Table 1: first, the UWB antenna using a copper sheet (CUWB), which has a thickness of $1 \mathrm{oz}$; second, the transparent radiator only UWB antenna (TRUWB) using a transparent semicircular radiator, which is composed of an IZTO/Ag/IZTO multilayer electrode with a copper sheet ground plane and microstripline; and, third, an entirely transparent UWB antenna (TUWB). Fabricated antennas are pictured in Figure 2. As shown in the figure, CUWB is composed of a copper sheet and is not transparent at all; however, the TRUWB is partially transparent, and the TUWB is entirely transparent.
TABLE 1: Composition of proposed antennas.

\begin{tabular}{lcc}
\hline & Semicircular radiator & Ground plane and microstripline \\
\hline CUWB & Copper sheet & Copper sheet \\
TRUWB & IZTO/Ag/IZTO & Copper sheet \\
TUWB & IZTO/Ag/IZTO & IZTO/Ag/IZTO \\
\hline
\end{tabular}

TABle 2: Process conditions.

\begin{tabular}{lcc}
\hline & IZTO & $\mathrm{Ag}$ \\
\hline Power supply & RF magnetron & DC magnetron \\
Power & $100 \mathrm{~W}$ & $10 \mathrm{~W}$ \\
Initial vacuum & $5 \times 10^{-7}$ Torr & $5 \times 10^{-7}$ Torr \\
Process vacuum & $3.5 \mathrm{mTorr}$ & $5 \mathrm{mTorr}$ \\
Oxygen partial pressure & $3 \%$ & - \\
Thickness & $45 \mathrm{~nm}$ & $10 \mathrm{~nm}$ \\
\hline
\end{tabular}

\section{Fabrication}

The IZTO/Ag/IZTO multilayer electrode is deposited on the acryl substrate. The thickness of IZTO is $45 \mathrm{~nm}$ and that of $\mathrm{Ag}$ is $10 \mathrm{~nm}$. IZTO and Ag are both deposited by the physical vapor deposition (PVD) process. The order of the fabrication is shown in Figure 3. Firstly, a mask is made to cover the nondeposited position. This step is called masking. Before deposition, a pure vacuum condition is established under $5 \times 10^{-7}$ Torr for pure samples. Then, an IZTO/Ag/IZTO multilayer is deposited using the PVD process. Next, the masking is removed. Likewise, the transparent ground plane is made using the previous order one more time. IZTO and $\mathrm{Ag}$ are grown by using different conditions of deposition.

Table 2 depicts the process conditions of the IZTO and Ag layers. IZTO and Ag are deposited in the initial vacuum under $5 \times 10^{-7}$ Torr using an RF magnetron and DC magnetron power supply, respectively. Then, $100 \mathrm{~W}$ of power is used for the IZTO layer in the vacuum of $3.5 \mathrm{mTorr}$ argon with 


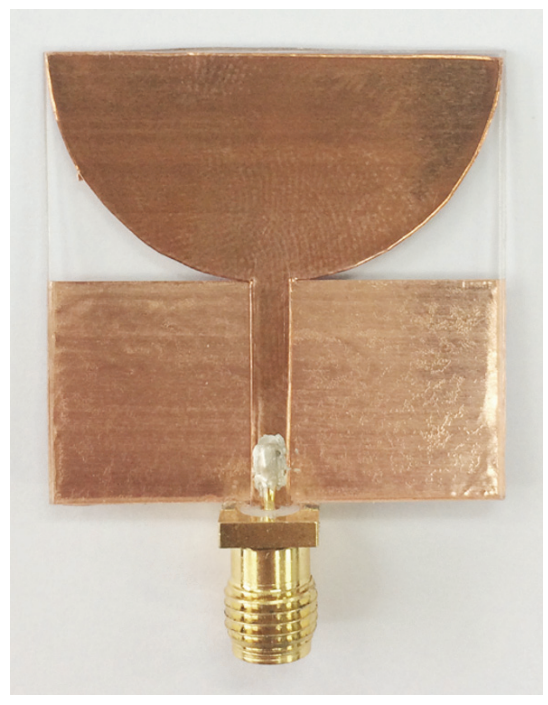

(a)

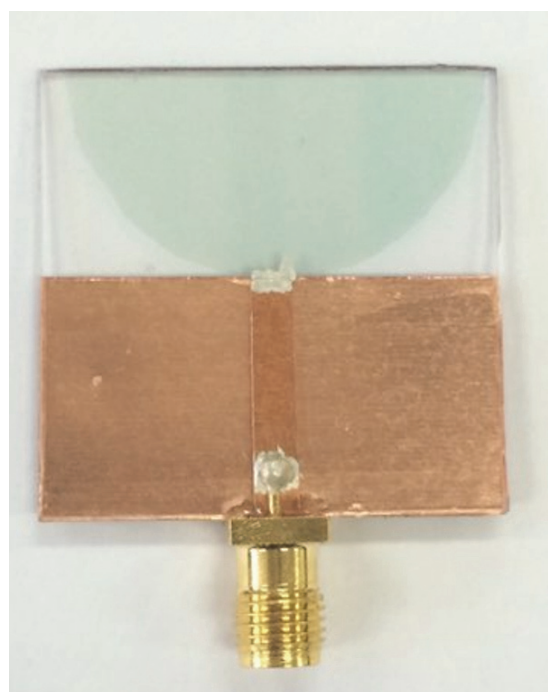

(b)

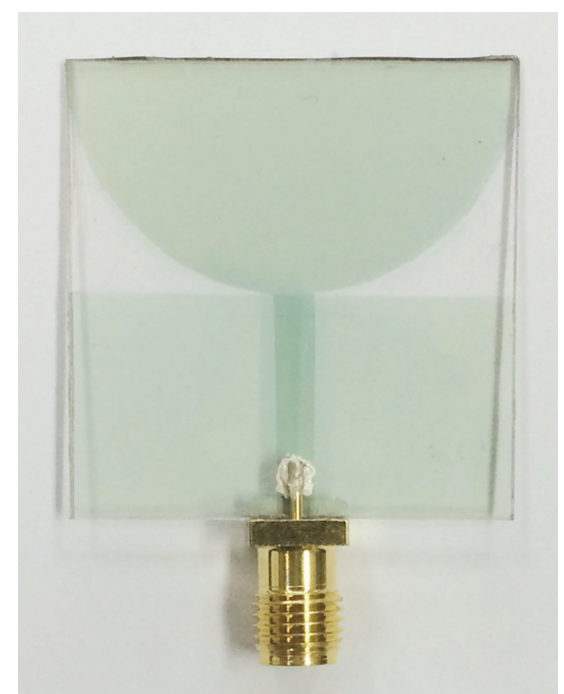

(c)

FIGURE 2: Fabricated antennas with copper sheet and transparent conductive films. (a) CUWB. (b) TRUWB. (c) TUWB.

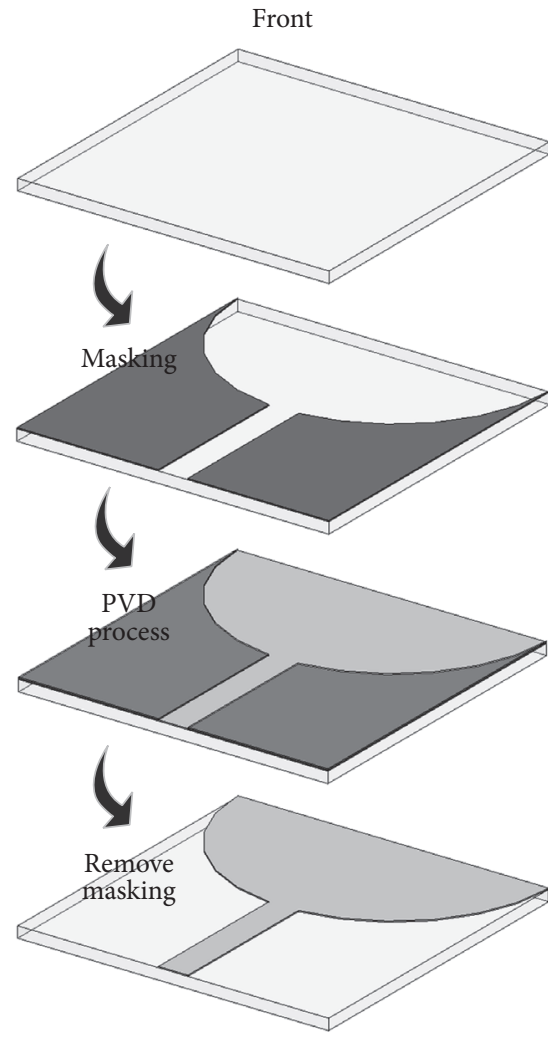

(a)

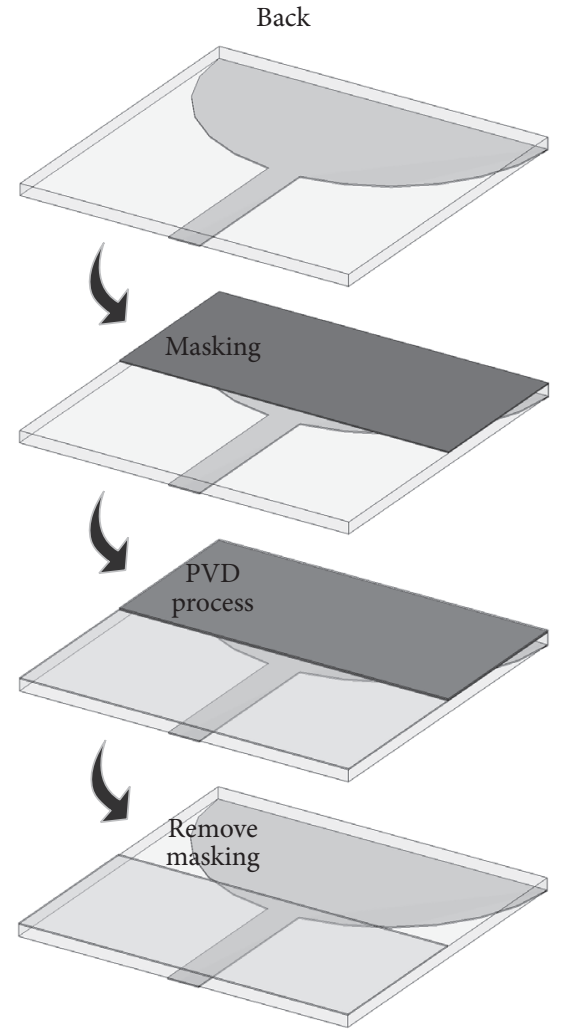

(b)

FIGURE 3: The fabrication process of the transparent UWB antenna. (a) Front. (b) Back. 


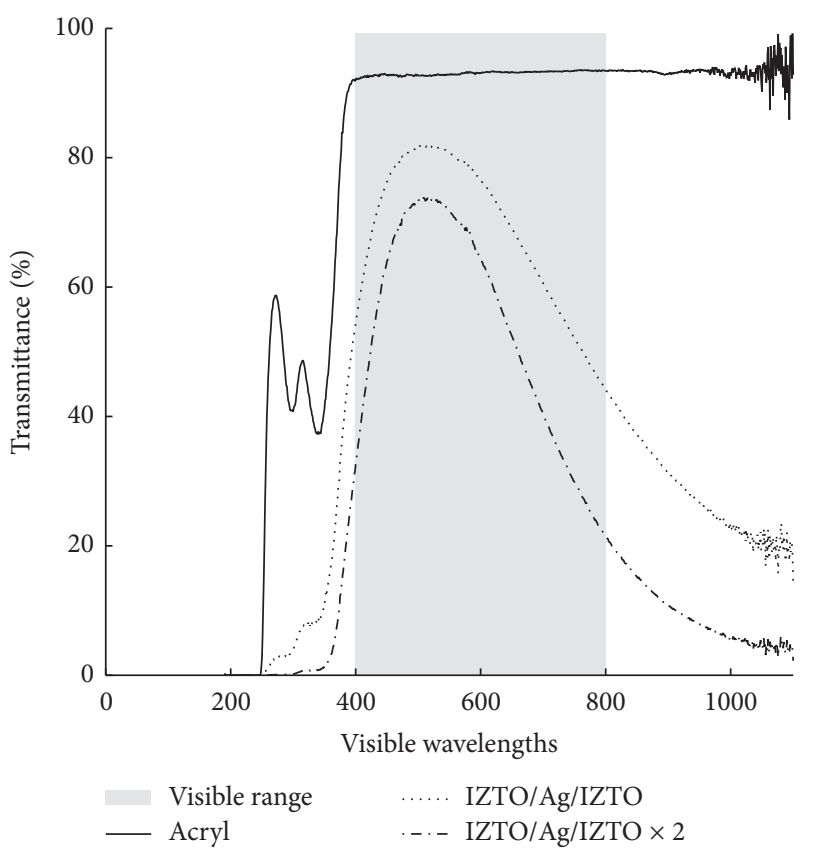

FIGURE 4: Transmittance of the transparent conductive film on the acryl substrate.

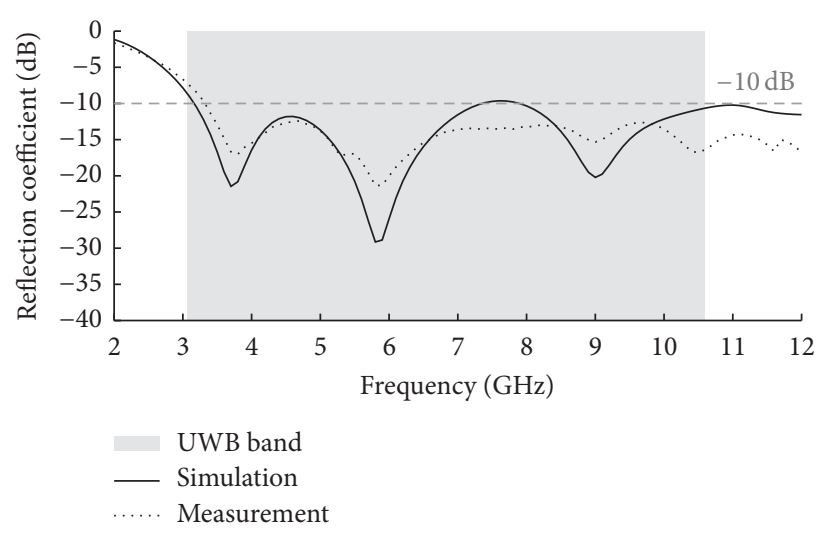

(a)

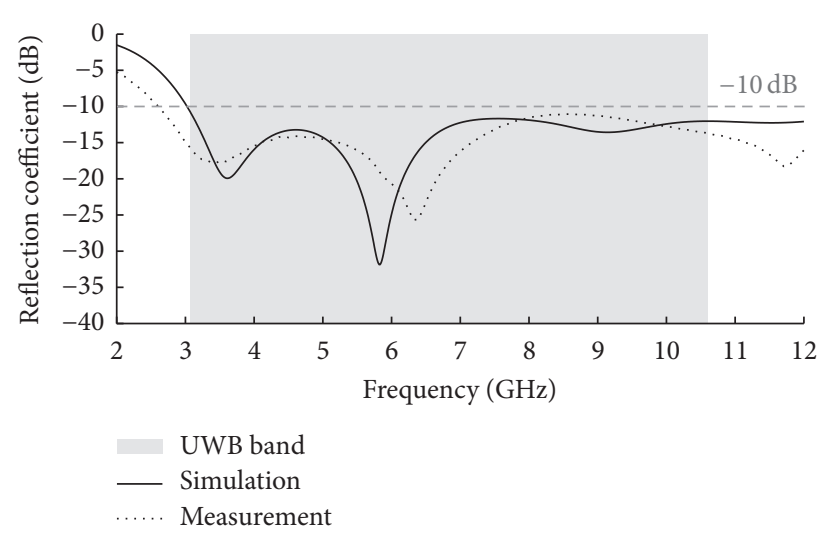

(b)

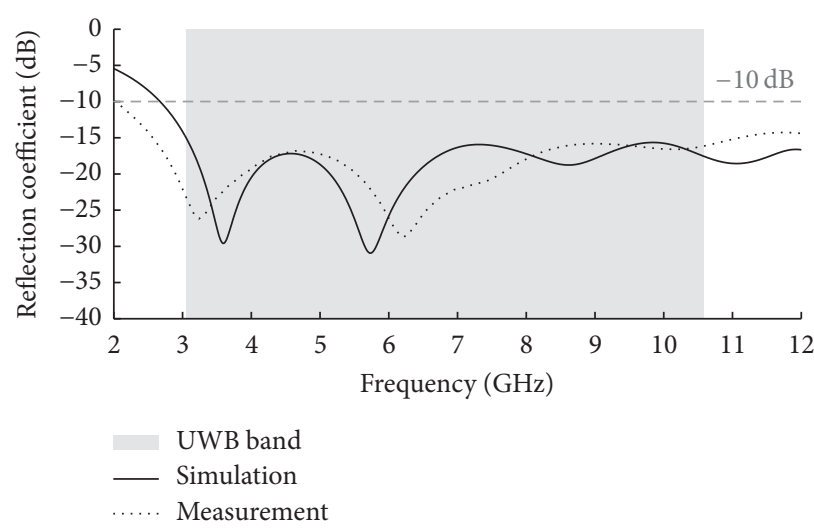

(c)

FIGURE 5: The reflection coefficient of transparent UWB antennas. (a) CUWB. (b) TRUWB. (c) TUWB. 

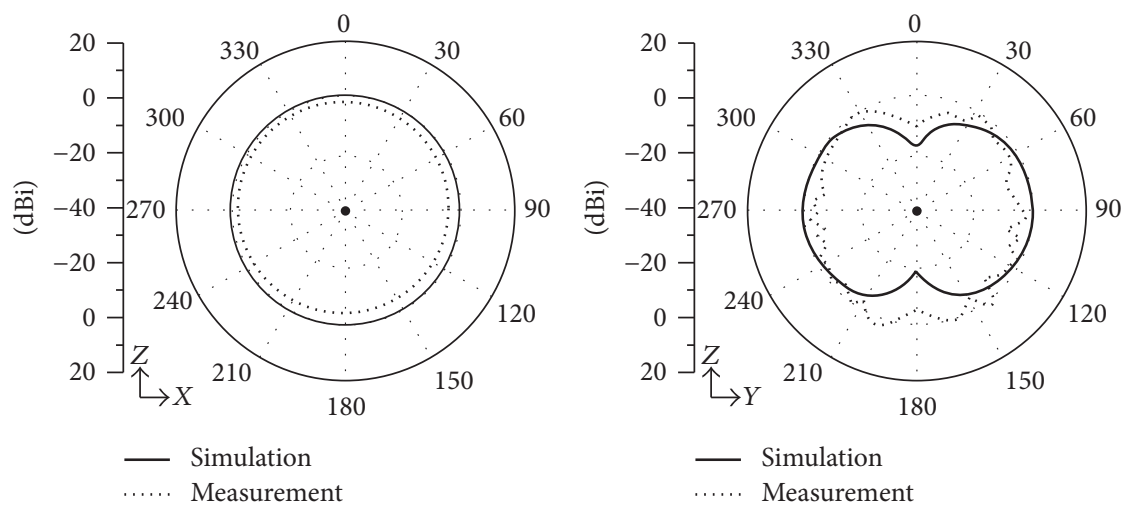

(a)
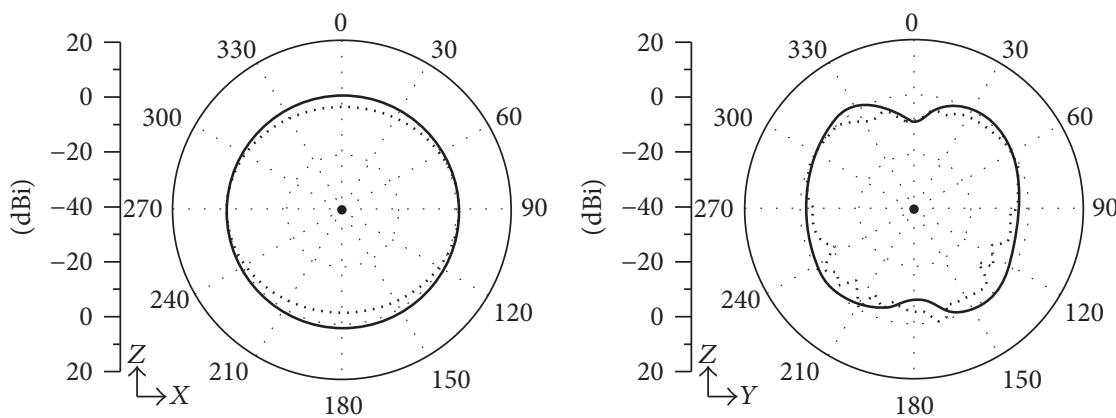

_..... Simulation

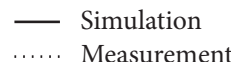

(b)
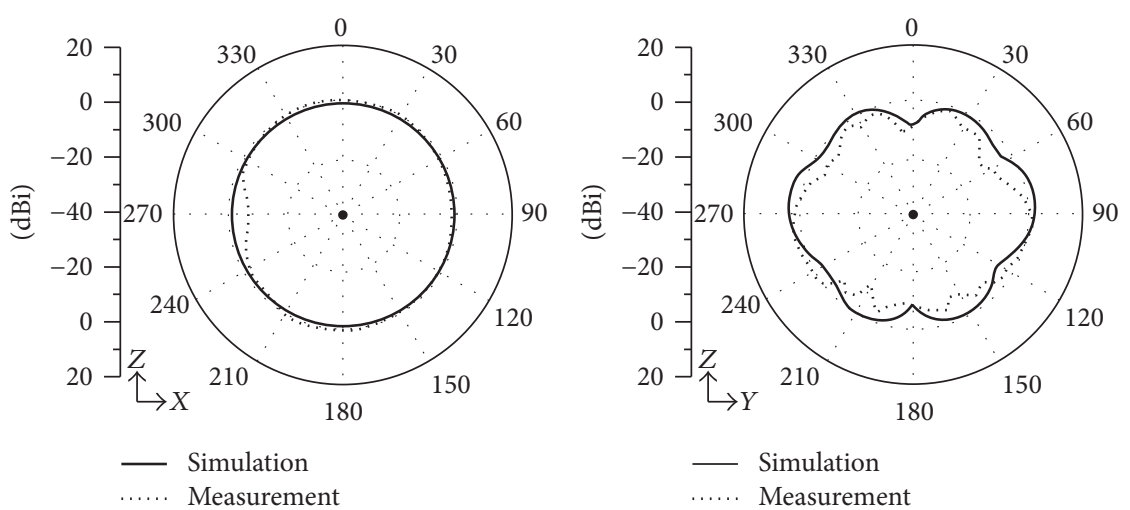

(c)
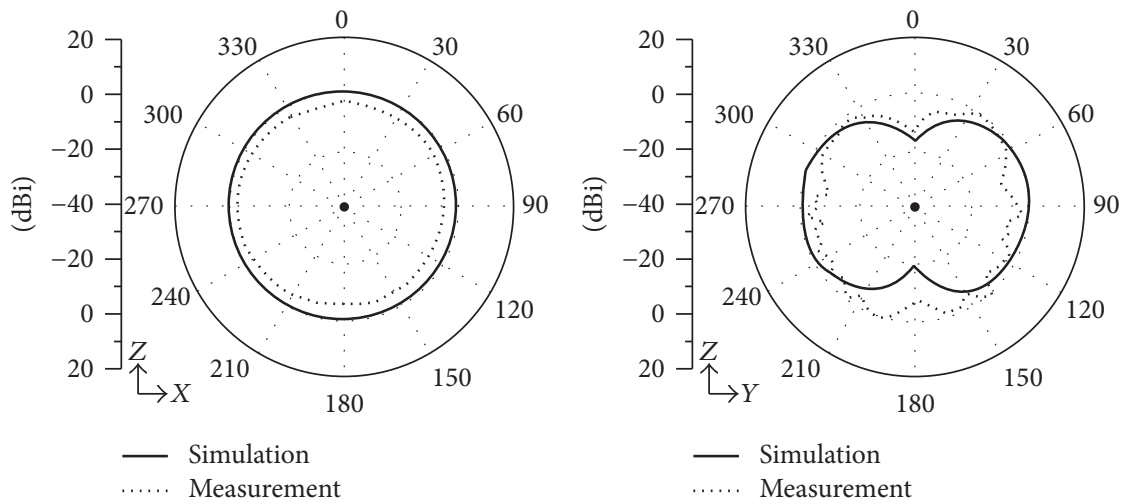

(d)

Figure 6: Continued. 

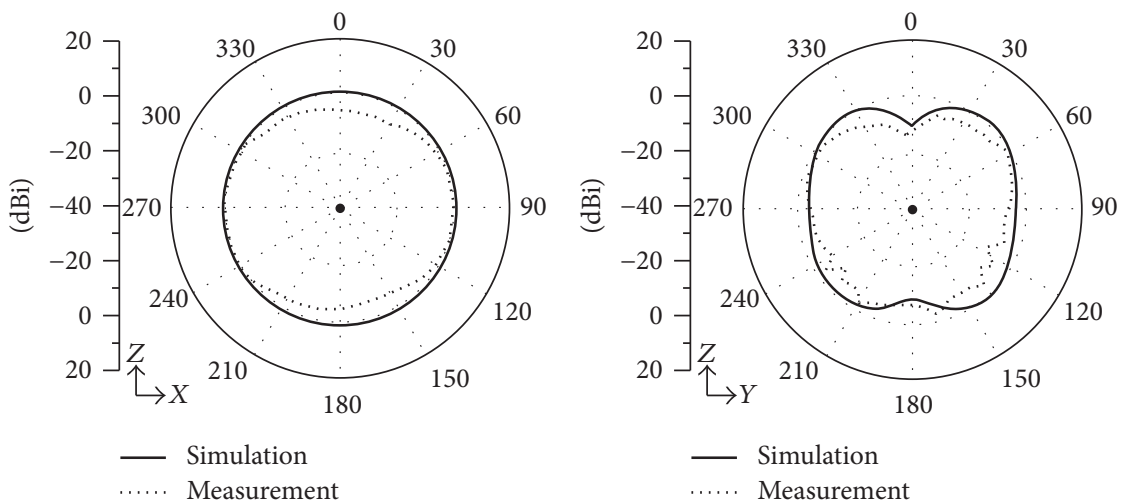

(e)
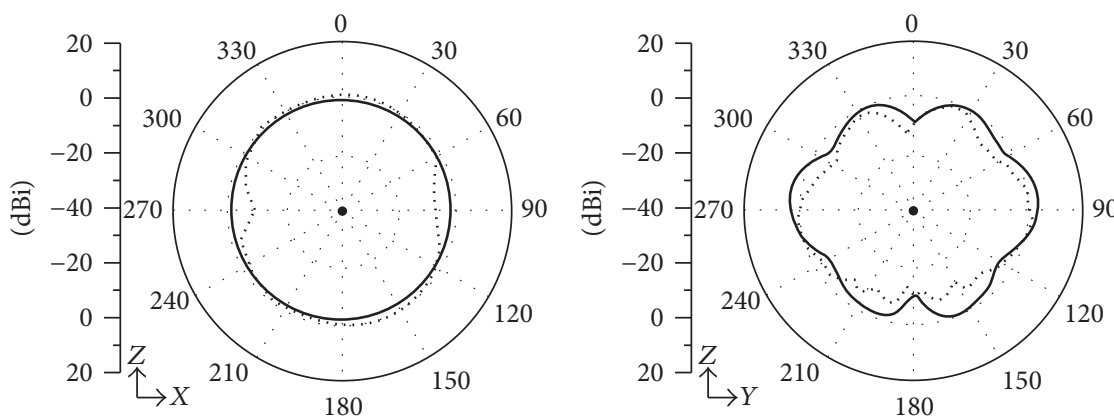

- Simulation

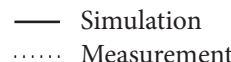

(f)
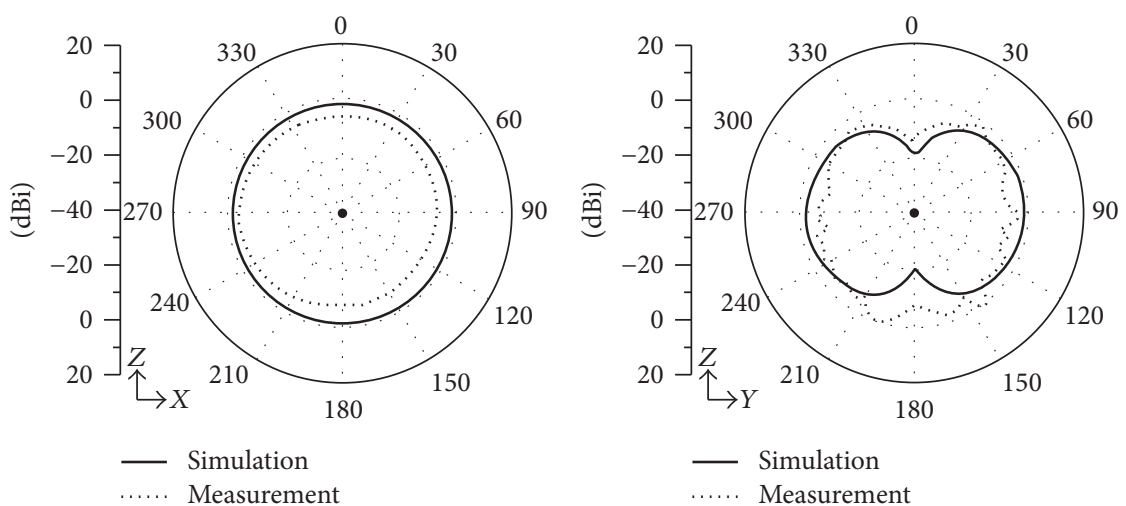

(g)
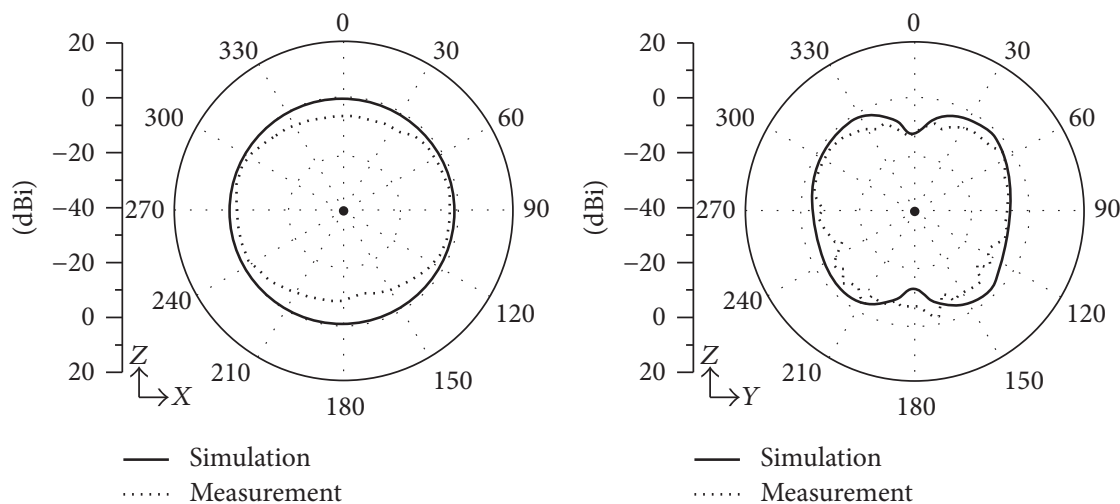

(h)

Figure 6: Continued. 

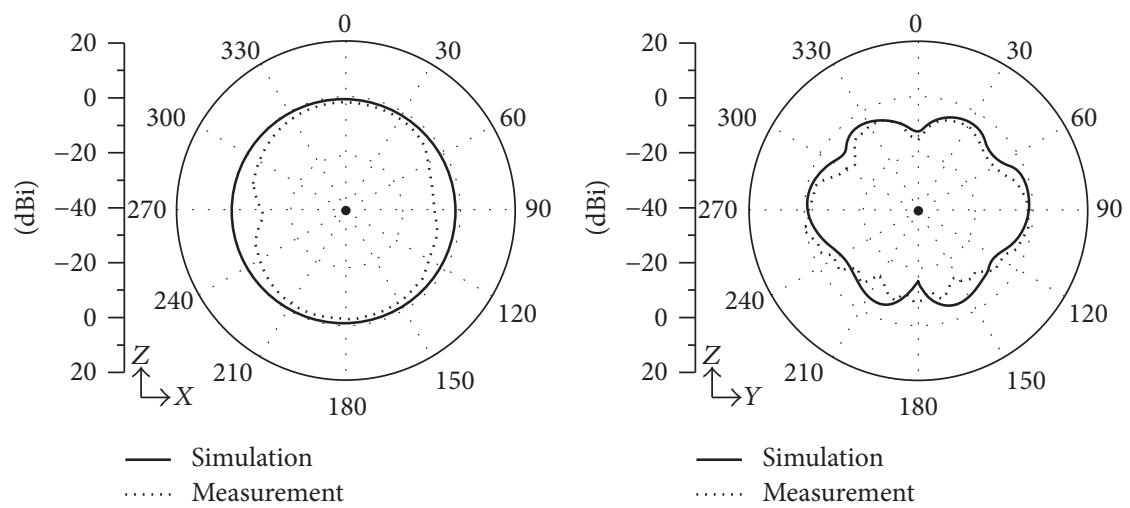

(i)

Figure 6: Simulated and measured $X Z$ and $Y Z$ planes of antenna radiation patterns. (a) CUWB at 3.1 GHz. (b) CUWB at 6.5 GHz. (c) CUWB at 10.6 GHz. (d) TRUWB at 3.1 GHz. (e) TRUWB at $6.5 \mathrm{GHz}$. (f) TRUWB at 10.6 GHz. (g) TUWB at 3.1 GHz. (h) TUWB at 6.5 GHz. (i) TUWB at $10.6 \mathrm{GHz}$.

an oxygen partial pressure of $3 \%$, and $10 \mathrm{~W}$ of power is used for the Ag layer in the vacuum of 5 mTorr argon. For the measurement of thickness, Tencor Alpha-Step 200 profilometer is used.

The transmittance and conductivity of the fabricated TCF are measured by a UV-visible spectrometer (SCINCO, S-3100) and a Hall effect measurement system (ECOPIA, HMS-3000), respectively. The transmittance is depicted in Figure 4. The transparent antennas on the acryl substrate have transmittance over $80 \%$ at $550 \mathrm{~nm}$ wavelength, which is required for the transparent displays [11]. The overlapped feeding line's transparency is IZTO/Ag/IZTO $\times 2$. The average transmittance in the visible range of wavelength from $400 \mathrm{~nm}$ to $800 \mathrm{~nm}$ is $68.63 \%$. The conductivity of the IZTO/Ag/IZTO multilayer electrode is $3,960,000 \mathrm{~S} / \mathrm{m}$. For the performance comparison of the transparent conductive film, Haacke suggested figure of merit (FOM) value [14]. Value of figure of merit (FOM) is determined by transmittance and sheet resistance of material. The proposed IZTO/Ag/IZTO electrode has highest optical transmittance at the point of $550 \mathrm{~nm}$ wavelength and has sheet resistance of $2.52 \Omega /$ sq. FOM is 0.3205 which is more conductive material than typical ITO (sheet resistance is $10 \Omega / \mathrm{sq}$ and transmittance is $80 \%$ ) [15].

\section{Simulation and Measurement Results}

The proposed antennas are simulated by a high frequency structure simulator (HFSS). The reflection coefficients of the fabricated antennas are shown in Figure 5. The band width of the UWB antennas is from $3.1 \mathrm{GHz}$ to $10.6 \mathrm{GHz}$. Reflection coefficients of the proposed antennas satisfy the band width of the UWB antennas under $-10 \mathrm{~dB}$. This is in good agreement with simulated and measured results, except a light difference that is mainly caused by the fabrication.

The antenna radiation pattern, peak gain, and efficiency are measured in the antenna anechoic chamber covered with a radio frequency (RF) absorber. The simulated and measured radiation patterns in the $X Z$ plane ( $H$-plane) and $Y Z$ plane (E-plane) at several frequency points $(3.1,6.5$,
TABLE 3: Efficiency and peak gain of the proposed antennas.

\begin{tabular}{lcccccc}
\hline \multirow{2}{*}{ Frequency $(\mathrm{GHz})$} & \multicolumn{3}{c}{ Efficiency $(\%)$} & \multicolumn{3}{c}{ Peak gain $(\mathrm{dBi})$} \\
& 3.1 & 6.5 & 10.6 & 3.1 & 6.5 & 10.6 \\
\hline CUWB & 64.9 & 84.8 & 81.7 & 4.12 & 4.17 & 4.16 \\
TRUWB & 52.2 & 64.5 & 78.4 & 2.69 & 3.14 & 2.76 \\
TUWB & 33.9 & 42.1 & 40.8 & 0.73 & 0.74 & 0.94 \\
\hline
\end{tabular}

and $10.6 \mathrm{GHz}$ ) are illustrated in Figure 6. The proposed antennas are omnidirectional. The radiation patterns of the CUWB, TRUWB, and TUWB are similar because of the same dimensions and design. There is good agreement of the radiation pattern with simulation and measurement results. The radiation patterns of the proposed antennas follow those of omnidirectional antennas. Radiation patterns at the 6.5 and $10.6 \mathrm{GHz}$ show harmonics.

The efficiencies and peak gains at several frequencies are tabulated in Table 3. At $3.1 \mathrm{GHz}$, the efficiencies of the proposed antennas of CUWB, TRUWB, and TUWB are 64.9, 52.2 , and $33.9 \%$ and peak gains are $4.12,2.69$, and $0.73 \mathrm{dBi}$; and at $6.5 \mathrm{GHz}$, the efficiencies are $84.8,64.5$, and $42.1 \%$ and peak gains are $4.17,3.14$, and $0.74 \mathrm{dBi}$, respectively. The efficiency is higher in the relatively higher frequencies [13]. As expected, CUWB has the highest efficiency and peak gain at the measured frequencies. Nevertheless, transparent UWB antennas have efficiencies over $33.9 \%$. These results show that IZTO/Ag/IZTO multilayer electrodes are applicable for transparent UWB antennas.

\section{Conclusion}

We have presented transparent UWB antennas using IZTO/ $\mathrm{Ag} / \mathrm{IZTO}$ multilayer electrodes that have transparency and conductivity over $80 \%$ at $550 \mathrm{~nm}$ wavelength and 3,960,000 S/m. There are two types of transparent antennas for each application. An UWB antenna with a transparent radiator (TRUWB) is designed for transparent devices using a copper ground plane and microstripline, and an entirely transparent UWB 
antenna (TUWB) is designed for entirely transparent devices. The results show that transparent antennas (TRUWB and TUWB) have lower efficiency than UWB antennas with copper sheets (CUWB). However, the proposed antennas have higher efficiency in the relatively higher frequencies [13], and TRUWB has higher efficiency than TUWB. In addition, the proposed transparent antennas satisfy reflection coefficients under $-10 \mathrm{~dB}$ in the range of UWB. Transparent omnidirectional antennas have higher efficiency than transparent directional antennas, such as a microstrip patch antenna, when it is made of transparent conducting oxides (TCOs) $[10,16]$. Studies for transparent devices will contribute to the development of technology. In the near future, transparent antennas will be used in the transparent part of devices for wireless applications.

\section{Competing Interests}

The authors declare that they have no competing interests.

\section{Acknowledgments}

This work is supported by a National Research Foundation (NRF) of Korea Grant funded by the Korean government (no. 2016R1D1A1B02012957).

\section{References}

[1] D. S. Ginley and C. Bright, "Transparent conducting oxides," MRS Bulletin, vol. 25, pp. 15-18, 2000.

[2] S. Hong, J. Yeo, G. Kim et al., "Nonvacuum, maskless fabrication of a flexible metal grid transparent conductor by lowtemperature selective laser sintering of nanoparticle ink," ACS Nano, vol. 7, no. 6, pp. 5024-5031, 2013.

[3] D. C. Paine, T. Whitson, D. Janiac, R. Beresford, C. O. Yang, and B. Lewis, "A study of low temperature crystallization of amorphous thin film indium-tin-oxide," Journal of Applied Physics, vol. 85, no. 12, pp. 8445-8450, 1999.

[4] T. Yasin, R. Baktur, and C. Furse, "A study on the efficiency of transparent patch antennas designed from conductive oxide films," in Proceedings of the IEEE International Symposium on Antennas and Propagation and USNC/URSI National Radio Science Meeting (APSURSI '11), pp. 3085-3087, July 2011.

[5] U. Betz, M. K. Olsson, J. Marthy, M. F. Escolá, and F. Atamny, "Thin films engineering of indium tin oxide: large area flat panel displays application," Surface and Coatings Technology, vol. 200, no. 20-21, pp. 5751-5759, 2006.

[6] K. Alzoubi, M. M. Hamasha, S. Lu, and B. Sammakia, "Bending fatigue study of sputtered ITO on flexible substrate," Journal of Display Technology, vol. 7, no. 11, pp. 593-600, 2011.

[7] S. Cattarin, M. Musiani, B. Tribollet, and V. Vivier, "Impedance response of resistive ITO electrodes," Journal of the Electrochemical Society, vol. 158, no. 7, pp. F135-F141, 2011.

[8] J. C. Bernède, L. Cattin, M. Morsli, and Y. Berredjem, "Ultrathin metal layer passivation of the transparent conductive anode in organic solar cells," Solar Energy Materials and Solar Cells, vol. 92, no. 11, pp. 1508-1515, 2008.

[9] Y. Kim, C. Lee, S. Hong, C. W. Jung, and Y. Kim, "Design of transparent multilayer film antenna for wireless communication," Electronics Letters, vol. 51, no. 1, pp. 12-14, 2015.
[10] C. M. Lee, Y. Kim, Y. Kim, I. K. Kim, and C. W. Jung, "A flexible and transparent antenna on a polyamide substrate for laptop computers," Microwave and Optical Technology Letters, vol. 57, no. 5, pp. 1038-1042, 2015.

[11] K.-H. Choi, H.-J. Nam, J.-A. Jeong et al., "Highly flexible and transparent $\mathrm{InZnSnO}_{x} / \mathrm{Ag} / \mathrm{InZnSnO}_{x}$ multilayer electrode for flexible organic light emitting diodes," Applied Physics Letters, vol. 92, no. 22, Article ID 223302, 2008.

[12] C. W. Jung and Y. Kim, "A half-moon antenna with tilt angles for wideband applications," Microwave and Optical Technology Letters, vol. 49, no. 9, pp. 2171-2174, 2007.

[13] J. R. Saberin and C. Furse, "Challenges with optically transparent patch antennas," IEEE Antennas and Propagation Magazine, vol. 54, no. 3, pp. 10-16, 2012.

[14] G. Haacke, "New figure of merit for transparent conductors," Journal of Applied Physics, vol. 47, no. 9, pp. 4086-4089, 1976.

[15] J.-Y. Lee, S. T. Connor, Y. Cui, and P. Peumans, "Solutionprocessed metal nanowire mesh transparent electrodes," Nano Letters, vol. 8, no. 2, pp. 689-692, 2008.

[16] S. Sheikh, M. Shokooh-Saremi, and M.-M. Bagheri-Mohagheghi, "Transparent microstrip antenna made of fluorine doped tin oxide: a comprehensive study," Journal of Electromagnetic Waves and Applications, vol. 29, no. 12, pp. 1557-1569, 2015. 


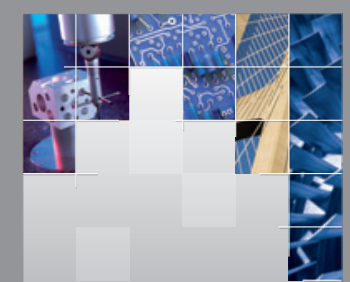

\section{Enfincering}
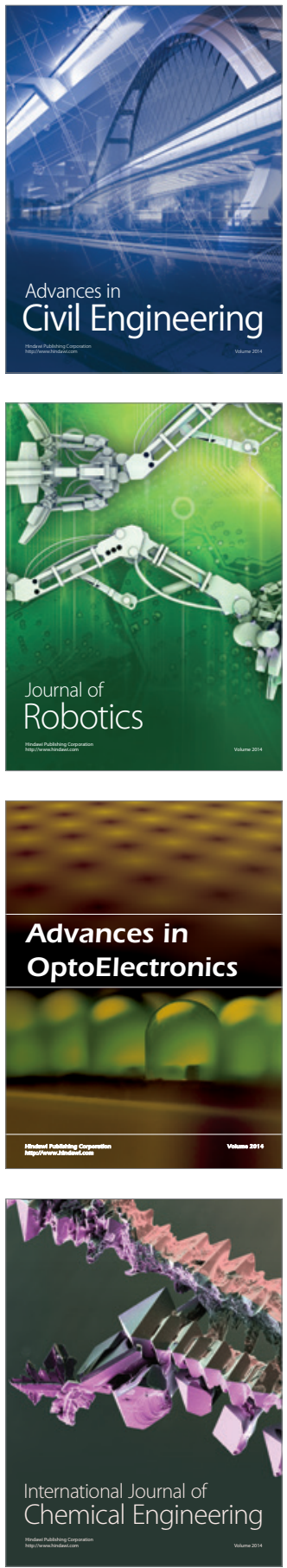

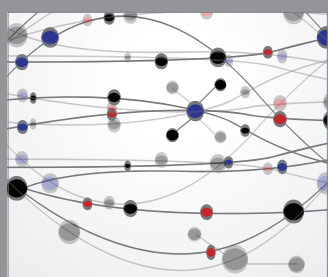

The Scientific World Journal

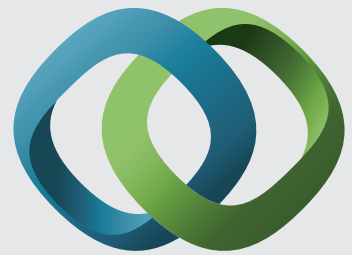

\section{Hindawi}

Submit your manuscripts at

http://www.hindawi.com
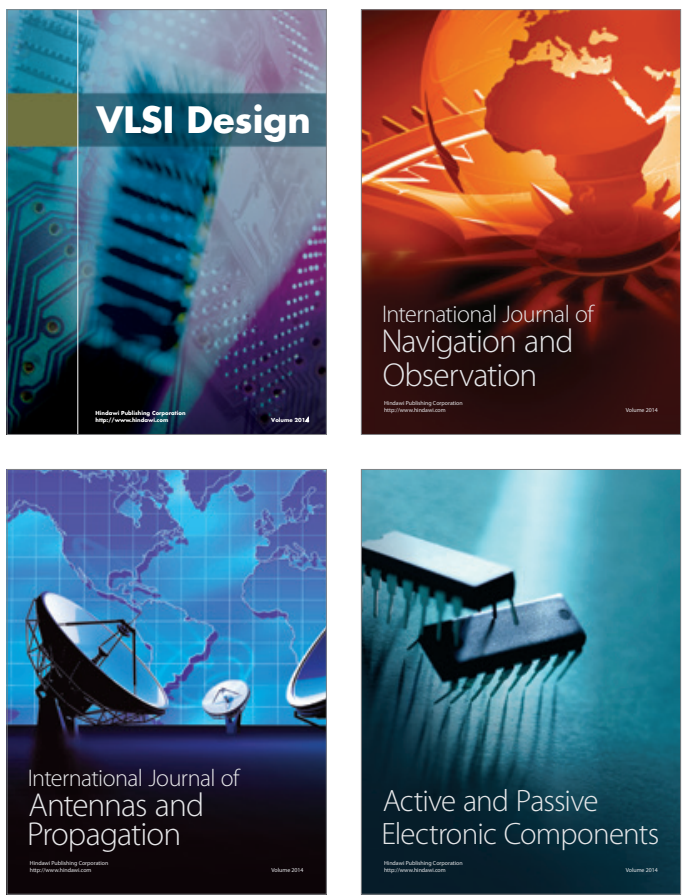
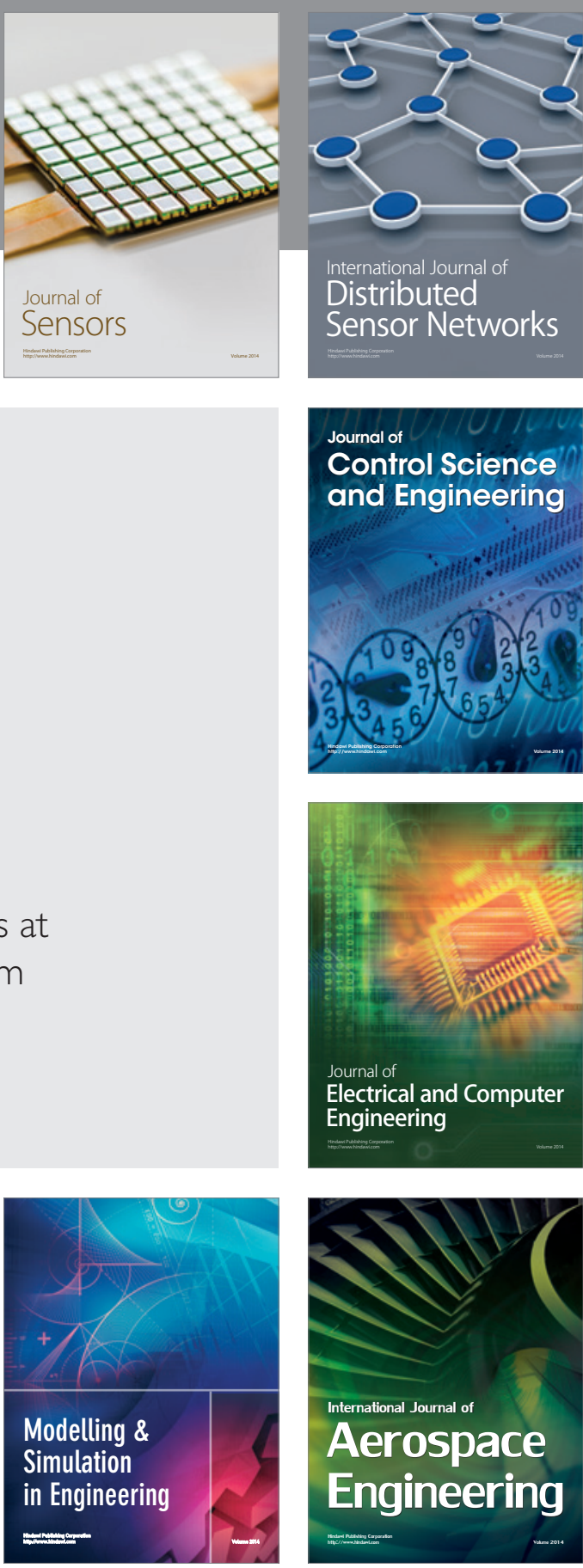

International Journal of

Distributed

Sensor Networks

Journal of

Control Science

and Engineering
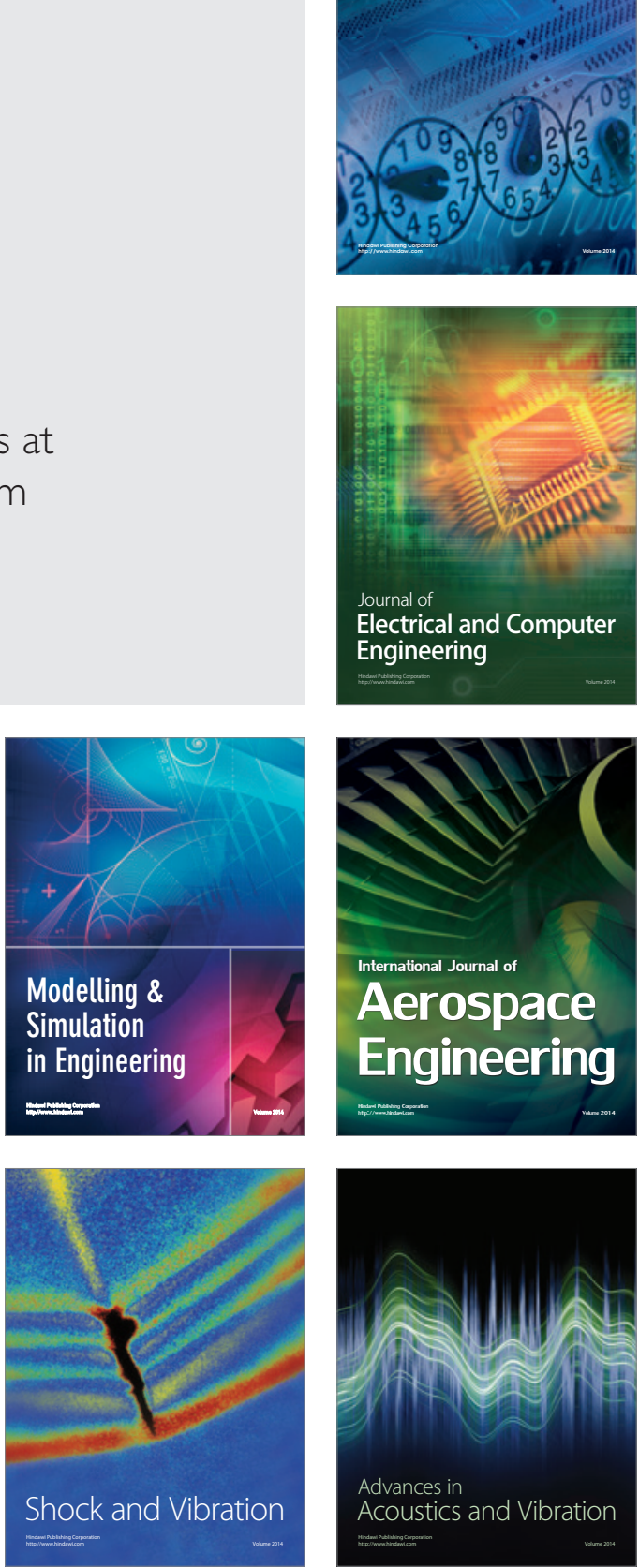